\title{
BMJ Open Sleep Treatment Outcome Predictors (STOP) Pilot Study: a protocol for a randomised controlled trial examining predictors of change of insomnia symptoms and associated traits following cognitive-behavioural therapy for insomnia in an unselected sample
}

Dan Denis, ${ }^{1,2}$ Thalia C Eley, ${ }^{2}$ Fruhling Rijsdijk, ${ }^{2}$ Helena M S Zavos, ${ }^{2}$ Robert Keers, ${ }^{3}$ Colin A Espie, ${ }^{4,5}$ Annemarie I Luik, ${ }^{4,5}$ Isabella Badini, ${ }^{6}$ Sarah Derveeuw, ${ }^{2}$ Alvin Romero, ${ }^{7}$ John Hodsoll, ${ }^{8}$ Alice M Gregory ${ }^{6}$

To cite: Denis D, Eley TC, Rijsdijk F, et al. Sleep Treatment Outcome Predictors (STOP) Pilot Study: a protocol for a randomised controlled trial examining predictors of change of insomnia symptoms and associated traits following cognitive-behavioural therapy for insomnia in an unselected sample. BMJ Open 2017;7:e017177. doi:10.1136/ bmjopen-2017-017177

- Prepublication history and additional material for this paper are available online. To view these files, please visit the journal online (http://dx.doi. org/10.1136/bmjopen-2017017177).

Received 5 April 2017

Revised 6 September 2017

Accepted 25 October 2017

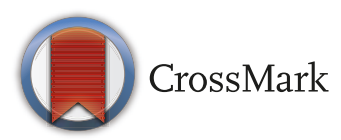

For numbered affiliations see end of article.

Correspondence to

Dr Alice M Gregory;

a.gregory@gold.ac.uk

\section{ABSTRACT}

Introduction Cognitive-behavioural therapy for insomnia (CBT-I) leads to insomnia symptom improvements in a substantial proportion of patients. However, not everyone responds well to this treatment, and it is unclear what determines individual differences in response. The broader aim of this work is to examine to what extent response to CBT-I is due to genetic and environmental factors. The purpose of this pilot study is to examine feasibility of a design to test hypotheses focusing on an unselected sample, that is, without selection on insomnia complaints, in order to plan a larger behavioural genetics study where most participants will likely not have an insomnia disorder. Methods and analysis A two parallel-group randomised controlled trial is being conducted across three London universities. Female students (minimum age 18 years) enrolled on a psychology programme at one of the three sites were invited to participate. The target number of participants to be recruited is 240 . Following baseline assessments, participants were randomly allocated to either the treatment group, where they received weekly sessions of digital CBT-I for 6 weeks, or the control group, where they completed an online puzzle each week for 6 weeks. Follow-up assessments have taken place midintervention (3 weeks) and end of intervention (6 weeks). A 6-month follow-up assessment will also occur. Primary outcomes will be assessed using descriptive statistics and effect size estimates for intervention effects. Secondary outcomes will be analysed using multivariate generalised estimating equation models.

Ethics and dissemination The study received ethical approval from the Research Ethics and Integrity subcommittee, Goldsmiths, University of London (application reference: EA 1305). DNA sample collection for the BioResource received ethical approval from the NRES

\section{Strengths and limitations of this study}

- This study contains a large sample size for a pilot study and will provide valuable effect size information useful for the planning of future investigations of this topic.

- Stratification on sleep problems was implemented to ensure baseline sleep problems were equal in both groups

- Recruitment was done using convenience sampling, which may lead to some self-selection bias in the sample.

- The online nature of the study makes it difficult to fully assess adherence to the intervention.

Committee South Central-0xford (reference number: 15/ $\mathrm{SC} / 0388$ ). The results of this work shall be published in peer-reviewed journals.

Trial registration number NCT03062891; Results.

\section{INTRODUCTION}

Insomnia occurs frequently and causes a substantial burden to society. ${ }^{1}$ It is estimated that as many as one-third of US adults experience issues with their sleep, and the annual cost of insomnia to the US labour force has been estimated to be approximately US $\$ 280$ billion. $^{2}$ Historically, insomnia has been considered as secondary to other psychiatric disorders, such as depression. ${ }^{3}{ }^{4}$ More recently, however, it has become clear that insomnia is associated with a wide range of 
psychiatric conditions and may precede and predict their development and severity. ${ }^{5-7}$

Cognitive-behavioural therapy (CBT) has been shown to be an effective treatment for insomnia. ${ }^{89}$ Consequently, the American College of Physicians now recommends CBT for insomnia (CBT-I) as the first-choice treatment for chronic insomnia. ${ }^{10}$ CBT-I is now more accessible than ever due to the development of automated online programmes, which have shown promising effectiveness. ${ }^{11-13}$ A randomised controlled trial of 164 participants meeting diagnostic and statistical manual of mental disorers, 5th edition (DSM-5) criteria for insomnia disorder showed significant post-treatment improvements in insomnia symptoms and sleep efficiency for those participants assigned to a digital CBT-I group compared with a placebo. ${ }^{11}$ These results were largely maintained at an 8-week follow-up. Following this, a meta-analysis of 15 randomised controlled trials investigating digital CBT-I found across all studies a significant improvement in sleep efficiency $(7.2 \%)$ following digital CBT-I compared with baseline and a significant drop on the Insomnia Severity Index, bringing patients to a subthreshold level of insomnia. ${ }^{13}$ This reduction in insomnia symptom severity was also accompanied by a significant drop in symptoms of depression, suggesting CBT-I may also be effective for problems associated with insomnia. ${ }^{14}$

Despite the demonstrated effectiveness of CBT-I, some individuals fail to respond to treatment. It has been suggested that CBT-I can significantly reduce symptoms of insomnia in around $70 \%$ of patients, meaning $30 \%$ of patients show no improvement in symptoms. ${ }^{15}$ Understanding the reasons why people either respond or do not respond to treatment holds promise of improving or tailoring current treatments for insomnia.

Investigations of predictors of treatment response to CBT applied to other conditions, such as anxiety, have shown a wide range of demographic, clinical, genetic and epigenetic factors influence response to CBT treatment for anxiety. ${ }^{16-19}$ Genetic predictors of treatment outcome are still unclear however. While some studies have reported specific genetic markers for intervention outcome in disorders such as post-traumatic stress disorder, ${ }^{20}$ panic disorder ${ }^{21}$ and social anxiety disorder, ${ }^{22}$ these findings have not always been replicated. ${ }^{23}{ }^{24}$ While individually, any genetic predictor is likely to only explain a small proportion of variance in treatment outcome, understanding these multiple factors and their interactions may serve an important role in improving the outcome of therapy. The aim of this pilot study is to test the feasibility of running a larger-scale study of predictors of treatment outcome for CBT-I within a twin design, where not all participants will have insomnia.

\section{PRIMARY OBJECTIVES}

Sleep improvement after CBT-I in an unselected sample

To date, studies examining CBT-I have done so in the context of improving symptoms in patients diagnosed with insomnia disorder. ${ }^{11}$ However, we plan to include both participants with and without insomnia in the main study. In particular, as our future study is likely to focus on twin pairs, it is inevitable that at least some of the participants in that study will not have insomnia (eg, one participant may have insomnia but their co-twin might not). We are therefore interested to see the extent to which CBT-I has an effect in an unselected sample, that is, participants with and without an insomnia disorder. This pilot aims to establish the distributional properties of individual differences in change score on measures such as the Sleep Condition Indicator (SCI) and Pittsburgh Sleep Quality Index (PSQI) ${ }^{2526}$ as a result of the CBT-I intervention. The outcome of this will be used to assess the feasibility of running a larger behavioural genetics study in the future investigating genetic predictors of CBT-I outcome in an unselected sample. As we are primarily interested in change in insomnia symptoms, the SCI will be our main outcome measure for this objective (see the Measures section for detail).

\section{Participation rate and treatment acceptability}

The second aim of the study is to assess the feasibility of a digital CBT-I intervention study in a non-clinical group. For example, will participants without insomnia be willing to complete a 6 -week online programme aimed at improving sleep? As such, we will be closely monitoring participation (the proportion of participants who are willing to take part in the study) and drop-out rates (the percentage of participants who sign up to the study and drop out before the end of the study). We will also examine treatment acceptability. While psychological interventions to treat insomnia such as CBT-I have been rated highly by patients with regards to how acceptable they find the treatment, ${ }^{27} 28$ it is important for this study to investigate whether participants who do not necessarily have a sleep disorder find CBT-I an acceptable treatment. This will be assessed using an adapted version of the Treatment Acceptability Questionnaire $(\mathrm{TAQ})^{29}$; for more information, see the Measures section.

\section{SECONDARY AIMS}

\section{Factors predicting treatment outcome}

We will collect data on factors that may predict treatment outcome and will be able to use the data collected to estimate effect sizes for various predictors, which will be useful in power calculations to estimate the sample size for a larger future behavioural genetics study. Furthermore, by including these measures in the pilot study, it will allow us to assess the feasibility of administering a large battery of questionnaires to participants in addition to completing the digital CBT-I/puzzles.

Based on previous work into predictors of treatment outcome in CBT for anxiety, ${ }^{16-19}$ a wide range of demographic, clinical and genetic predictors such as potential single-nucleotide polymorphisms and polygenic risk scores of response to digital CBT-I will be investigated 
(see the Measures section for more details) for producing an estimated effect size that will be helpful in the planning of a larger study.

\section{Sleep quality and implications for associated variables}

Sleep quality is known to be associated with a number of other variables, such as anxiety and depression. ${ }^{30}$ As such, one outcome of improving sleep quality through CBT-I could be an improvement of symptoms in associated variables. For example, in a meta-analysis of digital CBT-I randomised controlled trial (RCT) studies, it was found that digital CBT-I significantly reduced depression severity. ${ }^{14}$ As such, digital CBT-I holds the promise of improving sleep problems themselves and the variables commonly associated with them.

Our focus here is to obtain an approximate effect size for any effect that can be used in designing a more substantial study, but we note that we may not have power to report significant effects in the context of this pilot work.

\section{EXPLORATORY AIMS OF THE PILOT STUDY \\ Genetic predictors of treatment outcome}

We will perform preliminary analysis on possible genetic predictors of digital CBT-I treatment outcome to help in the development of hypotheses for a larger genetics study in the future. The samples will be genotyped on the Psychiatric Genomics Consortium customised HumanCoreExome-24 V.1.1 beadchip from Illumina. This beadchip retains a genome-wide association study (GWAS) backbone, exome beadchip content and an additional $\sim 50 \mathrm{~K}$ psychiatric relevant variants. We will also perform exploratory investigations on the genetic data in relation to exploratory aim 8 (see below).

\section{Mechanisms mediating improvements in insomnia}

Assuming enough variation in appropriate measures, we will investigate whether factors such as pre-sleep arousal, cognitions about sleep, chronotype and specific sleep disturbances mediate CBT-I outcome in an unselected sample.

\section{Improvement in sleep paralysis episodes following CBT-I}

Sleep paralysis is an unusual but relatively common condition involving a period of inability to perform voluntary movements at either sleep onset or on awakening, ${ }^{31}$ with an estimated prevalence of up to $30 \% .{ }^{32}{ }^{33}$ If enough participants with sleep paralysis are included in our sample, we will assess the feasibility and effectiveness of digital CBT-I in the patient groups in terms of reducing the frequency of episodes, as well as associated fear and hallucinations.

\section{Variables associated with exploding head syndrome}

Exploding head syndrome is an unusual experience, characterised by hearing loud noises (eg, an explosion or gunshot) in one's head at either wake-sleep or sleepwake transitions. ${ }^{31}$ If enough participants with exploding head syndrome are included in our study, we shall look at potentially associated variables such as insomnia symptoms, stress and psychopathology.

\section{METHODS AND ANALYSIS}

\section{Study dates}

Recruitment for the study started November 2016, and data will have finished being collected by the end of September 2017. The study was retrospectively registered on 5 December 2016. The reason the trial was registered retrospectively was due to very restricted limitations on when participants could be recruited (see the Participant recruitment section). Unfortunately, the trial was not registered until after the first recruitment dates had passed. Rather than lose potential recruiting opportunities, we decided to register the trial retrospectively.

\section{Design}

The study was a two-group parallel randomised controlled trial in which the intervention group received a digital CBT-I intervention and the control group received a weekly online puzzle. See the Intervention section for more details.

Participants were female students (both undergraduate and postgraduate) completing a psychology programme at one of three London universities (for full details, see the trial registration). After completing the baseline assessment online via the Qualtrics system, participants were randomly allocated to either the CBT-I or puzzles group. Three weeks later, participants completed a second online assessment and then a third online assessment 6 weeks after the start of the study. Finally, a follow-up online assessment was carried out 6 months after group allocation. Participants were also invited to give a DNA sample at the start of data collection. While we have limited statistical power to look at genetic predictors of treatment outcomes in the pilot study, these samples could be pooled with other data collected in the future and also provide a useful opportunity for our collaborators to collect data for another ongoing research initiative. ${ }^{34}$ See figure 1 for a detailed outline of the study timeline. A completed Standard Protocol Items: Recommendations for Interventional Trials checklist and WHO trial registration data set can be found in online supplementary files 1 and 2.

\section{Inclusion and exclusion criteria}

Only women were eligible for participation. This is because the majority of the students on the psychology courses are women, and so adding men would create heterogeneity but without sufficient power to examine this further. Furthermore, only individuals enrolled in a psychology course from one of three London universities were recruited due to reasons of convenience. We focused our recruitment efforts on first year students in particular, as it is possible that a small number of students in other years may have already taken part in 


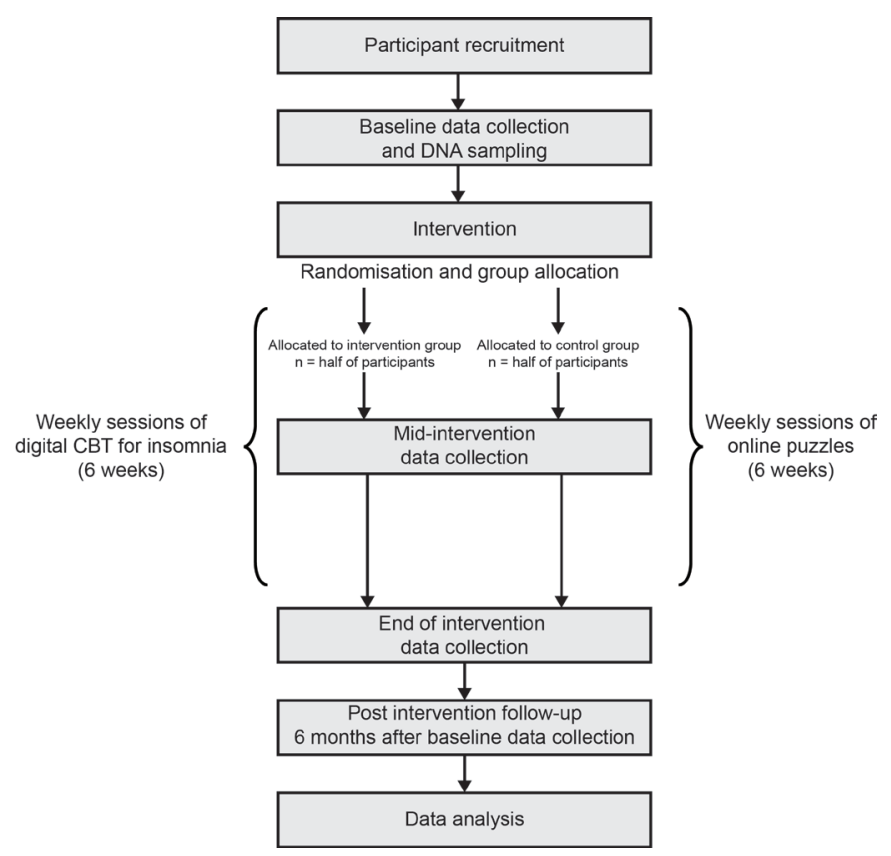

Figure 1 Flow chart of study timeline. CBT, cognitivebehavioural therapy.

studies using the same digital CBT-I platform. ${ }^{35}$ In order to address this point explicitly, in the questionnaires given to participants, they were asked if they have had any experience with Sleepio before taking part in this study.

\section{Participant recruitment}

Participants were recruited to the study using a two-step procedure. Initially, potential participants were contacted via an e-mail that provided the study information, specific instructions as to the nature of the recruitment day and contact information.

The second stage of recruitment involved a series of recruitment days at the three sites. These recruitment days were timed to coincide with classes that potential participants were present at, to make it more likely that they would be in university. At sign-up, participants were given a paper copy of the information sheet and were given the option to ask any questions about the nature of the study. After confirming that they were happy to take part in the study, all participants were asked to give informed consent, provide a DNA sample (see the DNA sample collection section) and were assigned a unique participant ID number, which was used for future assessments. To allow the participation of individuals who wished to take part in the study but were unable to sign up in person, participants were given the option to contact the research team directly by email in order to arrange providing consent to take part in the study.

Participants were rewarded for their time, either in the form of course credits (offered credits $+£ 5$ online gift voucher) or online shopping voucher (£40), awarded to them on completion of the study.

\section{Randomisation and study automation}

After collection of baseline data, participants were randomly allocated to either the CBT-I group or the puzzles group. A member of the research team randomised eligible participants using the blockrand package for $\mathrm{R}^{36}$ Participants were stratified based on age, sleep problems and study site. Stratification on age was performed to assure similar age distributions in both groups. Stratification on sleep problems was implemented to avoid the possibility of a disproportionate number of participants with sleep problems being randomly allocated to the same group. Stratification for study site was implemented to avoid an unnecessary delay between completing the first questionnaire and being allocated to a group.

An automated email was sent to participants to inform them of which group they had been assigned. Those in the CBT-I group were given further information as to the nature of the programme (see the Digital CBT-I section) as well as a unique code needed to $\log$ in to the website. Those in the puzzles group were given information as to the nature of the tasks that they were required to complete (see the Puzzles section). Participants were not able to change groups once they had been allocated.

\section{INTERVENTION}

\section{Digital CBT-I}

CBT-I participants received six weekly CBT-I sessions delivered by an animated 'virtual therapist' (The Prof) via the online platform 'Sleepio' (http://www.sleepio.com). The programme comprised a fully automated media-rich web application, driven dynamically by baseline, adherence, performance and progress data, and provides additional access to elements such as an online library with background information, a community of fellow users and support, prompts and reminders sent by email.

The Sleepio programme covers behavioural (eg, sleep restriction, stimulus control) and cognitive (eg, putting the day to rest, thought restructuring, imagery, articulatory suppression, paradoxical intention, mindfulness) strategies, as well as additional relaxation strategies (progressive muscle relaxation and autogenic training) and advice on lifestyle and bedroom factors (sleep hygiene). As part of the intervention, participants filled in a daily sleep diary. The intervention was based on a previously validated manual. ${ }^{37-39}$ Sleepio has been shown to improve sleep and associated daytime functioning in adults diagnosed with insomnia disorder. ${ }^{11}$

\section{Puzzles}

Participants in the control group were sent weekly puzzles to complete within Qualtrics. Each puzzle was designed to be cognitively engaging, and time taken to complete a puzzle was matched as closely as possible to the time taken to complete one session of digital CBT-I. Puzzles were sent directly to participants via automated distribution emails sent at 7-day intervals. In order to track whether participants were completing the puzzles, they 
were required to enter their participant ID number at the start of each puzzle. The types of puzzles administered to participants included word searches, crosswords and lateral thinking problems.

\section{DATA COLLECTION}

\section{DNA sample collection}

This project was conducted in collaboration with the National Institute for Health Research (NIHR) Biomedical Research Centre (BRC) BioResource for Mental and Neurological Health in London as part of a national NIHR initiative to build up a central library of information (or 'BioBank)' about people's health.

In this study, we obtained saliva samples from our participants after obtaining consent during the recruitment days. Samples were collected by a researcher from the BioResource team in compliance with their ethically approved protocol. The BRC is the custodian of the samples received. On receipt, samples were logged and prepared for extraction of DNA. The BRC ensured that genetic samples were processed in accordance with strict health and safety guidelines and under the requirements of the Human Tissue Act (HTA). King's College London holds a HTA licence, number: 12293. All samples are stored in tubes labelled with a barcode that includes the participant number. The link between the participant ID and de-identified data is kept in a secure folder. The DNA samples collected as part of this study are stored by the BRC for future analysis and hypothesis testing with appropriate ethical approval in the future, and under existing BRC BioResource approvals.

\section{Wave 1 data collection}

Eligible participants were given the option of completing the baseline survey online after signing up. Participants were encouraged to complete the survey within 1 week from sign-up. Paper copies were made available for participants who had problems with their device or internet access.

Participants completed all measures, as shown in table 1. Participants had the option to leave out any question. The survey took $30-40 \mathrm{~min}$ to complete. At the end the survey, participants were reminded that they would be contacted with regards to future data collection.

\section{Waves 2-4 data collection}

The second and third waves of data collection were carried out 3 weeks and 6 weeks following allocation. The fourth wave was carried out 6 months following the allocation of participants to groups. These time points corresponded to mid-intervention, end-of-intervention and post-intervention follow-up time points, respectively (see figure 1 for more detail). Automated emails distributed by Qualtrics were sent to participants at the designated intervals. Not all measures are assessed at all waves, as shown in table 1. Follow-up emails to non-responders were sent each week to participants who fall behind on their tasks (ie, CBT-I, puzzles or surveys).

\section{MEASURES}

Descriptions for all measures used are provided below. For waves 2-4, some measures were adapted to ask participants to consider their answers with reference to the last 2 weeks (unless otherwise stated below) in order to ensure participants were considering only the time since the last wave of data collection when responding. Full details on each measure used can be found in online supplementary file 3 .

Demographic information was collected at baseline. At the start of each survey, participants were asked to indicate whether it was currently term time, examination time or holiday time. At wave 2, participants in the Sleepio group indicated whether they had ever used Sleepio before.

\section{Sleep measures}

Insomnia symptoms-Sleep Condition Indicator. ${ }^{25}$ An eightitem measure assessing symptoms of insomnia used to identify insomnia symptoms in community samples. ${ }^{40}$

Sleep quality-Pittsburgh Sleep Quality Index.$^{26}$ An 18-item questionnaire assessing seven components of sleep quality and disturbances, which also yields a global score of sleep quality. The scale has been shown to be reliable and valid in assessing sleep quality in adult community samples. ${ }^{41}$

Trauma-related sleep disturbances-Pittsburgh Sleep Quality Index Addendum. ${ }^{42}$ Assesses frequency of seven sleep disturbances typically related to trauma. The measure has been validated for use in assessing these disturbances. ${ }^{42} 43$

Pre-sleep arousal-Pre-sleep Arousal Scale. ${ }^{44}$ Measures symptoms of cognitive (eight items) and somatic (eight items) arousal experienced around bedtime. It is has been validated with respect to objective measures of pre-sleep arousal. ${ }^{45} 46$

Cognitions about sleep-Dysfunctional Beliefs About Sleep Scale. ${ }^{47}$ A 10-item questionnaire that includes items about sleep-disruptive cognitions such as faulty beliefs, worry and attentional bias. The measure has shown to be reliable. $^{48}$

Chronotype-Munich Chronotype Questionnaire (MCTQ) ${ }^{49}$ Chronotype is estimated as the midpoint of sleep on workdays and work-free days minus half of the difference between sleep duration on work-free days and average sleep duration of the work to control for sleep debt (ie, the midpoint of sleep on work-free days, corrected for sleep duration). The MCTQ is a reliable and valid measure of chronotype..$^{51}$

Sleep paralysis-Waterloo Unusual Experiences Questionnaire (WUSEQ) ${ }^{52}$ Items from the WUSEQ were used to assess the frequency of sleep paralysis and associated hallucinations. The measure is valid and reliable in healthy student samples. ${ }^{53} 54$

Sleep paralysis-Fearful Isolated Sleep Paralysis Interview (FISPI) ${ }^{55}$ Two items from this measure were adapted to measure the amount of fear/distress typically caused by sleep paralysis episodes and how much interference with waking life episodes have caused. The FISPI has been used as a valid and reliable measure of sleep paralysis in university samples. ${ }^{56}$ 
Table 1 Schedule of enrolment, interventions and assessments made at each wave

\begin{tabular}{|c|c|c|c|c|c|c|c|}
\hline & Measures & Enrolment & $\begin{array}{l}\text { Wave } 1 \\
\text { baseline }\end{array}$ & Allocation & $\begin{array}{l}\text { Wave } 2 \\
\text { (3weeks) } \\
\text { mid-assessment }\end{array}$ & $\begin{array}{l}\text { Wave } 3 \\
\text { (6 weeks) } \\
\text { end assessment }\end{array}$ & $\begin{array}{l}\text { Wave } 4 \\
\text { (6 months) } \\
\text { follow-up }\end{array}$ \\
\hline \multicolumn{8}{|l|}{ Enrolment } \\
\hline Eligibility screening & & $\mathrm{x}$ & & & & & \\
\hline Informed consent & & $\mathrm{x}$ & & & & & \\
\hline Saliva DNA sample & & $x$ & & & & & \\
\hline Allocation & & & & $x$ & & & \\
\hline \multicolumn{8}{|l|}{ Interventions } \\
\hline CBT-I & & & & & $x$ & $x$ & \\
\hline Puzzles & & & & & $\mathrm{x}$ & $x$ & \\
\hline \multicolumn{8}{|l|}{ Assessments } \\
\hline Demographics & & & $\mathrm{x}$ & & & & \\
\hline Medical history & & & $x$ & & & & \\
\hline Weight and height & & & $\mathrm{x}$ & & & $x$ & $x$ \\
\hline Time of year & & & $x$ & & $x$ & $x$ & $x$ \\
\hline \multirow[t]{9}{*}{ Sleep measures } & $\mathrm{SCl}$ & & $x$ & & $x$ & $x$ & $x$ \\
\hline & PSQI & & $x$ & & $\mathrm{x}$ & $x$ & $x$ \\
\hline & PSQI-A & & $\mathrm{x}$ & & & $\mathrm{x}$ & $\mathrm{x}$ \\
\hline & PSAS & & $x$ & & $x$ & $x$ & $x$ \\
\hline & DBAS & & $x$ & & $\mathrm{x}$ & $x$ & $x$ \\
\hline & MCTQ & & $x$ & & . & $x$ & $x$ \\
\hline & WUSEQ & & $\mathrm{x}$ & & & $x$ & $x$ \\
\hline & FISPI & & $x$ & & & $x$ & $x$ \\
\hline & MUPS & & $x$ & & & & \\
\hline \multirow[t]{7}{*}{ Well-being measures } & STAI & & $x$ & & $x$ & $x$ & $x$ \\
\hline & MFQ & & $x$ & & $x$ & $x$ & $x$ \\
\hline & ADHD & & $\mathrm{x}$ & & & $x$ & $x$ \\
\hline & SPEQ & & $\mathrm{x}$ & & & $x$ & $x$ \\
\hline & PMH & & $x$ & & $x$ & $x$ & $x$ \\
\hline & PSS & & $x$ & & $x$ & $x$ & $x$ \\
\hline & LTE & & $x$ & & $x$ & $x$ & $x$ \\
\hline \multirow[t]{5}{*}{ Lifestyle measures } & $\begin{array}{l}\text { Sleeping } \\
\text { arrangements }\end{array}$ & & $\mathrm{x}$ & & $\mathrm{x}$ & $\mathrm{x}$ & $\mathrm{x}$ \\
\hline & Alcohol intake & & $x$ & & $x$ & $x$ & $x$ \\
\hline & $\begin{array}{l}\text { Caffeine } \\
\text { intake }\end{array}$ & & $\mathrm{x}$ & & $x$ & $\mathrm{x}$ & $x$ \\
\hline & $\begin{array}{l}\text { Smoking } \\
\text { behaviour }\end{array}$ & & $x$ & & & & \\
\hline & $\begin{array}{l}\text { Vaping } \\
\text { behaviour }\end{array}$ & & $\mathrm{x}$ & & & & \\
\hline $\begin{array}{l}\text { Treatment } \\
\text { acceptability }\end{array}$ & $\mathrm{TAQ}^{*}$ & & & & $x$ & $\mathrm{x}$ & \\
\hline
\end{tabular}

*Only administered to the Sleepio group.

ADHD, attention deficit hyperactivity disorder; CBT-I, cognitive-behavioural therapy for insomnia; DBAS, Dysfunctional Beliefs About Sleep Scale; FISPI, Fearful Isolated Sleep Paralysis Interview; LTE, List of Threatening Experiences; MCTQ, Munich Chronotype Questionnaire; MFQ, Moods and Feelings Questionnaire; MUPS, Munich Parasomnia Screening; PMH, Positive Mental Health Scale; PSAS, Pre-sleep Arousal Scale; PSQI, Pittsburgh Sleep Quality Index; PSQI-A, Pittsburgh Sleep Quality Index Addendum; PSS, Perceived Stress Scale; SCI, Sleep Condition Indicator; SPEQ, Specific Psychotic Experiences Questionnaire (paranoia, hallucinations and cognitive disorganisation subscales); STAI, State-Trait Anxiety Index; TAQ, Treatment Acceptability Questionnaire; WUSEQ, Waterloo Unusual Experiences Scale. 
Exploding head syndrome-Munich Parasomnia Screening (MUPS).${ }^{57}$ Lifetime prevalence of exploding head syndrome was measured using a single item from the MUPS.

\section{Psychopathology and well-being measures}

Anxiety symptoms-State-Trait Anxiety Index (STAI) ${ }^{58}$ The STAI assesses both state (20 items) and trait (20 items) levels of anxiety, and is a valid and reliable measure of anxiety symptoms. ${ }^{59}$

Depressed mood-Mood and Feelings Questionnaire (MFQ) ${ }^{60}$ Depressed mood was measured using the 13-item MFQ. This has been shown to be a valid measure of depressed mood.

Attention deficit hyperactivity disorder (ADHD) symptomatology-Bespoke measure examined 18 symptoms of ADHD according to DSM- 5 criteria. ${ }^{61}$ This is a valid and reliable measure of ADHD symptoms, and has been previously used in young adults to assess ADHD symptomatology in the context of sleep quality. ${ }^{62}$

Psychotic experiences-Specific Psychotic Experiences Questionnaire. ${ }^{63}$ Subscales relating to paranoia, ${ }^{64}$ hallucinations ${ }^{65}$ and cognitive disorganisation ${ }^{66}$ were used as they are strongly related with sleep disturbances. ${ }^{67}$ The scale has been shown to have good reliability and validity. ${ }^{63}$

Positive mental health-Positive Mental Health Scale. ${ }^{68}$ Positive aspects of health and life experiences were assessed using a nine-item questionnaire.

Life stress-Perceived Stress Scale (PSS) ${ }^{69}$ Life stress was measured with a 10-item measure. A review of articles assessing the psychometric properties of the PSS found the measure to be a reliable and valid measure of life stress. ${ }^{70}$

Exposure to threatening events-List of Threatening Experiences (LTE) ${ }^{71}{ }^{72}$ Participants were asked to indicate whether they had experienced any threatening events from a list of 24. The LTE has been shown to have high reliability and be a valid measure of exposure to potentially threatening experiences. ${ }^{73}$

\section{Lifestyle measures}

At each wave, participants were asked about their sleeping arrangements, ${ }^{74}$ and alcohol ${ }^{75}$ and caffeine intake. ${ }^{76}$ Cigarette $^{75}$ and electronic cigarette usage $\mathrm{e}^{77-79}$ were assessed at baseline.

\section{Treatment acceptability}

The six-item Treatment Acceptability Questionnaire (TAQ) ${ }^{29}$ asked specific questions regarding the degree to which they found the treatment acceptable, ethical and effective. There were also specific questions about the nature of the virtual therapist. Only participants in the Sleepio group received the TAQ.

\section{Sample size}

For this study, the target was to have 200 participants, which should provide power to examining our primary research questions, though we plan to over-recruit to account for some attrition throughout the study. As such, 240 participants will be recruited. Power analyses are often conducted using hypothesised effect sizes based on mean differences (eg, before and after treatment). However, as this is a pilot for a future behavioural genetics study, the main statistic of interest is not mean differences but individual differences (ie, variances). The decision to recruit 200 participants for this pilot study was mainly based on personal experiences of recruiting undergraduates from our institutions.

\section{STATISTICAL ANALYSIS}

\section{Primary objectives}

CBT-I in an unselected sample

The aim is to examine variation in response to CBT-I (ie, variation in the change score of self-reported insomnia symptoms, as measured by the SCI). To this end, we will obtain an effect size for the difference in change scores between the two groups on the SCI scale. Previous RCTs using the SCI as an outcome measure have observed a large effect size (Cohen's $d=1.50$ ) when comparing baseline score with post-treatment score. ${ }^{11}$ It is possible in our sample the effect size will be smaller, given the fact that participants will not necessarily meet insomnia criteria. Nevertheless, a small effect is still expected.

We will then look at differences between groups, by comparing the percentage of participants who finish with SCI scores in different ranges. We will also look at how many participants score below and above the suggested cut-off score for probable insomnia symptoms. Previous data suggest a cut-off of 16 , with a score below that meaning probable symptoms of insomnia. ${ }^{25}$ Furthermore, we will calculate the percentage of participants in the digital CBT-I group that will be above the mean score of the control group (Cohen's $\mathrm{U}_{3}$ ), the percentage of the two groups that overlap and the probability that one person picked at random from the digital CBT-I group will have a higher score than a person picked at random from the control group (the probability of superiority) ${ }^{80}$

\section{Participation rate and treatment acceptability}

Evaluation of participation rate and treatment acceptability will be based on the descriptive statistics, that is, percentage of participants who sign up to the study and complete it, the percentage from each group who drop out at each stage and mean scores on treatment acceptability questionnaire. Ninety-five per cent CIs for participation rate and acceptability scores will be calculated, which will show the upper and lower bound values of where the true population parameter will appear. Formal tests will be conducted to compare participant rates between the two groups ( $\chi^{2}$ analysis). For treatment acceptability, $\chi^{2}$ tests will test the proportion of participants selecting each response option.

\section{Secondary objectives}

Factors predicting treatment outcome and sleep quality and implications for associated variables

We will test which measures at baseline are moderators of longitudinal outcome of change scores in insomnia symptoms with multivariate generalised estimating equation 
models using Akaike/Bayesian information criteria to select an optimal model with predictors of insomnia symptoms/ sleep quality derived. All models will be run in Stata and control for covariates (eg, age) and the non-independence of sibling-pair data. Missing data shall be accounted for using maximum likelihood or multiple imputation procedures. Due to the small sample size, power may not be sufficient to investigate interaction effects. However, they shall be performed as an exploratory analysis.

\section{Ethics, consent, confidentiality and data security}

All stages of the study received ethical approval from the Research Ethics and Integrity subcommittee at Goldsmiths, University of London (application reference: EA 1305). DNA sample collection for the BioResource received ethical approval from the NRES Committee South Central-Oxford (reference number: 15/SC/0388). All participants were asked to provide informed consent before participating. It was made explicit that participation in the study is voluntary, that participants could choose not to answer questions if they did not want to, that they had the right to withdraw from the study at any point and that their data would remain confidential. Participants were informed of the intention to publish results from this study using their data and agreed to this in the informed consent. Copies of questionnaire booklets given to participants are not publically available due to copyright restrictions on some of the measures.

All identifying information was stored in a password-protected document. Survey responses were automatically stored in Qualtrics. No identifying information was stored with response data. Data in Qualtrics are secured using industry best standards (https://www.qualtrics.com/ security-statement/). At the end of data collection, datafiles for each wave of the study shall be downloaded off of Qualtric's servers and stored in SPSS. At this point, the datafiles will be removed from Qualtrics. Only researchers directly involved in the analysis of data will have access to participant data.

\section{Dissemination of findings}

Results of this trial will be disseminated primarily via peer-reviewed journal publications. It is expected that the primary, secondary and exploratory aims 5 and 6 of this study will be reported in a single publication. Other findings of exploratory aims will be published separately. The results of this study will also be available on the ClinicalTrials.gov website when they become available.

\section{Strengths and weaknesses of the protocol}

A key strength of the study was the use of an online CBT-I intervention. The online feature of this intervention is important as it provides easy access for participants. Furthermore, by using an automated system, there was no need for participants to interact with a CBT therapist during the intervention. This made it a more efficient programme than face-to-face CBT, which also meant that it was more feasible to administer to a large sample. It also meant that everyone received the same experience.
Examining treatment acceptability for digital CBT-I in a non-selected sample represents a novel investigation that will yield important findings for future researchers wishing to look at this intervention in non-clinical populations. Exploration of potential mechanisms underlying changes in insomnia symptoms is also a strong aspect of this study, as it will contribute to our knowledge of how CBT-I works in reducing symptoms.

Weaknesses include selection bias in the sample. It is possible that those who already suffer from sleep problems (despite not necessarily having an insomnia disorder) were more likely to take part in the study, although recruitment emails emphasised that participants did not have to suffer from any sleep problems to take part. Our convenience sample was also not a representative one, meaning that it may be hard to generalise findings to other populations. Relatedly, it is conceivable that psychology students, as compared with others, may react differently and rate the effectiveness differently to a psychological therapy.

\section{Judging study success}

When considering whether the study will be successful (ie, what results will suggest that a larger, behavioural genetics study is warranted), multiple variables will be considered. These will be the participation rates, treatment acceptability and effect size. As everything will be looked at together, strict criteria will not be set.

\section{Author affiliations}

${ }^{1}$ Department of Psychiatry, Beth Israel Deaconess Medical Center, Harvard Medical School, Boston, MA, USA

${ }^{2}$ MRC Social, Genetic, and Developmental Psychiatry Centre, Institute of Psychiatry, Psychology, and Neuroscience, King's College London, London, UK

${ }^{3}$ School of Biological and Chemical Sciences, Queen Mary University of London, London, UK

${ }^{4}$ Sleep and Circadian Neuroscience Institute, Nuffield Department of Clinical Neurosciences, University of Oxford, Oxford, UK

${ }^{5}$ Big Health Ltd, London, UK

${ }^{6}$ Department of Psychology, Goldsmiths, University of London, London, UK

${ }^{7}$ SLaM BioResource for Mental Health, South London and Maudsley NHS Foundation

Trust, King's College London, London, UK

${ }^{8}$ Department of Biostatistics, Institute of Psychiatry, Psychology, and Neuroscience, King's College London, London, UK

Acknowledgements We would like to thank Kimberley Goldsmith for her assistance in designing key parts of this study.

Contributors Study conception and design: TCE, FR, HMSZ, RK, CAE, JH, AMG. Acquisition of data: DD, HMSZ, RK, AIL, IB, SD, AR, AMG. Future analysis of data: DD, FR, SD. Preparation of manuscript: all authors.

Funding The study represents independent research funded by the National Institute for Health Research (NIHR) Biomedical Research Centre (BRC) at South London and Maudsley NHS Foundation Trust and King's College London.

Disclaimer The views expressed are those of the authors and not necessarily those of the NHS, the NIHR or the Department of Health. The funder had no role in the study design; collection, management, analysis and interpretation of data; writing of this protocol or subsequent publications; or in the decision to submit any future reports for publication.

Competing interests The position of AlL at the University of Oxford is funded by Big Health Ltd, the company behind the digital CBT-I programme evaluated in this study. CAE is the cofounder and CMO of Big Health Ltd and holds shares in Big Health Ltd. AMG has provided guidance and educational content for a freely available educational website focused on infant sleep. This website is partially 
supported by Johnson and Johnson, but they do not have any influence over content and do not advertise on it. AMG also contributes to BBC Focus Magazine.

Ethics approval Research Ethics and Integrity subcommittee, Goldsmiths, University of London and NRES Committee South Central—0xford.

Provenance and peer review Not commissioned; externally peer reviewed.

Open Access This is an Open Access article distributed in accordance with the terms of the Creative Commons Attribution (CC BY 4.0) license, which permits others to distribute, remix, adapt and build upon this work, for commercial use provided the original work is properly cited. See: http://creativecommons.org/ licenses/by/4.0/

(C) Article author(s) (or their employer(s) unless otherwise stated in the text of the article) 2017. All rights reserved. No commercial use is permitted unless otherwise expressly granted.

\section{REFERENCES}

1. Kessler RC, Berglund PA, Coulouvrat $\mathrm{C}$, et al. Insomnia and the performance of US workers: results from the America insomnia survey. Sleep 2011;34:1161-71

2. Hafner M, Stepanek M, Taylor J, et al. Why sleep matters - the economic costs of insufficient sleep: a cross-country comparative analysis. Rand Health Q 2017;6:11.

3. Katz DA, McHorney CA. Clinical correlates of insomnia in patients with chronic illness. Arch Intern Med 1998;158:1099-107.

4. Kupfer DJ. Pathophysiology and management of insomnia during depression. Ann Clin Psychiatry 1999;11:267-76.

5. Harvey AG. Insomnia: symptom or diagnosis? Clin Psychol Rev 2001;21:1037-59.

6. Baglioni C, Battagliese G, Feige B, et al. Insomnia as a predictor of depression: a meta-analytic evaluation of longitudinal epidemiological studies. J Affect Disord 2011;135:10-19.

7. Neckelmann D, Mykletun A, Dahl AA. Chronic insomnia as a risk factor for developing anxiety and depression. Sleep 2007;30:873-80.

8. Trauer JM, Qian MY, Doyle JS, et al. Cognitive behavioral therapy for chronic insomnia: a systematic review and meta-analysis. Ann Intern Med 2015;163:191-204.

9. van Straten A, van der Zweerde T, Kleiboer A, et al. Cognitive and behavioral therapies in the treatment of insomnia: a meta-analysis. Sleep Med Rev 2017.

10. Qaseem A, Kansagara D, Forciea MA, et al. Management of chronic insomnia disorder in adults: a clinical practice guideline from the American College of Physicians. Ann Intern Med 2016;165:125-33.

11. Espie CA, Kyle SD, Williams C, et al. A randomized, placebocontrolled trial of online cognitive behavioral therapy for chronic insomnia disorder delivered via an automated media-rich web application. Sleep 2012;35:769-81.

12. Zachariae R, Lyby MS, Ritterband LM, et al. Efficacy of internetdelivered cognitive-behavioral therapy for insomnia-a systematic review and meta-analysis of randomized controlled trials. Sleep Med Rev 2016;30:1-10.

13. Seyffert M, Lagisetty $P$, Landgraf $J$, et al. Internet-delivered cognitive behavioral therapy to treat insomnia: a systematic review and metaanalysis. PLoS One 2016;11:e0149139

14. Ye YY, Zhang YF, Chen J, et al. Internet-based cognitive behavioral therapy for insomnia (icbt-i) improves comorbid anxiety and depression-a meta-analysis of randomized controlled trials. PLoS One 2015;10:e0142258.

15. Morin CM. Cognitive-behavioral approaches to the treatment of insomnia. J Clin Psychiatry 2004;65(Suppl 16):33-40.

16. Eley TC, Hudson JL, Creswell C, et al. Therapygenetics: the 5HTTLPR and response to psychological therapy. Mol Psychiatry 2012;17:236-7.

17. Hudson JL, Lester KJ, Lewis CM, et al. Predicting outcomes following cognitive behaviour therapy in child anxiety disorders: the influence of genetic, demographic and clinical information. J Child Psychol Psychiatry 2013;54:1086-94.

18. Roberts S, Lester KJ, Hudson JL, et al. Serotonin transporter [corrected] methylation and response to cognitive behaviour therapy in children with anxiety disorders. Trans/ Psychiatry 2014;4:e444.

19. Hudson JL, Keers R, Roberts S, et al. Clinical predictors of response to cognitive-behavioral therapy in pediatric anxiety disorders: the Genes for Treatment (GxT) Study. J Am Acad Child Adolesc Psychiatry 2015;54:454-63.

20. Bryant RA, Felmingham KL, Falconer EM, et al. Preliminary evidence of the short allele of the serotonin transporter gene predicting poor response to cognitive behavior therapy in posttraumatic stress disorder. Biol Psychiatry 2010;67:1217-9.
21. Lonsdorf TB, Rück C, Bergström J, et al. The COMTval158met polymorphism is associated with symptom relief during exposurebased cognitive-behavioral treatment in panic disorder. BMC Psychiatry 2010:10:99.

22. Andersson E, Rück C, Lavebratt $C$, et al. Genetic polymorphisms in monoamine systems and outcome of cognitive behavior therapy for social anxiety disorder. PLoS One 2013;8:e79015.

23. Lester KJ, Roberts S, Keers R, et al. Non-replication of the association between 5HTTLPR and response to psychological therapy for child anxiety disorders. Br J Psychiatry 2016;208:182-8.

24. Bockting CL, Mocking RJ, Lok A, et al. Therapygenetics: the 5HTTLPR as a biomarker for response to psychological therapy? Mol Psychiatry 2013;18:744-5.

25. Espie CA, Kyle SD, Hames $\mathrm{P}$, et al. The sleep condition indicator: a clinical screening tool to evaluate insomnia disorder. BMJ Open 2014;4:e004183.

26. Buysse DJ, Reynolds CF, Monk TH, et al. The Pittsburgh sleep quality index: a new instrument for psychiatric practice and research. Psychiatry Res 1989;28:193-213.

27. Morin CM, Gaulier B, Barry T, et al. Patients' acceptance of psychological and pharmacological therapies for insomnia. Sleep 1992;15:302-5.

28. Vincent $\mathrm{N}$, Lionberg $\mathrm{C}$. Treatment preference and patient satisfaction in chronic insomnia. Sleep 2001;24:411-7.

29. Hunsley J. Development of the treatment acceptability questionnaire. J Psychopathol Behav Assess 1992;14:55-64.

30. Gregory AM, Buysse DJ, Willis TA, et al. Associations between sleep quality and anxiety and depression symptoms in a sample of young adult twins and siblings. J Psychosom Res 2011;71:250-5.

31. American Academy of Sleep Medicine. International classification of sleep disorders. 3rd edn. Darien, IL: Americian Academy of Sleep Medicine, 2014.

32. Denis D, French CC, Rowe R, et al. A twin and molecular genetics study of sleep paralysis and associated factors. J Sleep Res 2015;24:438-46.

33. Gorkana Surveys. Sleep paralysis. 2015 http://www.gorkanasurveys. com/C6WOXAACO (accessed 9 Mar 2017).

34. NIHR. BioResource for Mental Health. https://bioresource.nihr.ac.uk/ about-us/south-london-and-maudsley-2/ (accessed 31 Aug 2017).

35. Freeman D, Sheaves B, Goodwin GM, et al. Effects of cognitive behavioural therapy for insomnia on the mental health of university students: study protocol for a randomized controlled trial. Trials 2015;16:236.

36. Snow G. Blockrand: randomizationfor block random clinical trials. 2013.

37. Espie CA, Inglis SJ, Harvey L. Predicting clinically significant response to cognitive behavior therapy for chronic insomnia in general medical practice: analysis of outcome data at 12 months posttreatment. J Consult Clin Psychol 2001;69:58-66.

38. Espie CA, MacMahon KM, Kelly HL, et al. Randomized clinical effectiveness trial of nurse-administered small-group cognitive behavior therapy for persistent insomnia in general practice. Sleep 2007;30:574-84.

39. Espie CA, Fleming L, Cassidy J, et al. Randomized controlled clinical effectiveness trial of cognitive behavior therapy compared with treatment as usual for persistent insomnia in patients with cancer $J$ Clin Oncol 2008:26:4651-8.

40. Sheaves B, Porcheret K, Tsanas A, et al. Insomnia, nightmares, and chronotype as markers of risk for severe mental illness: results from a student population. Sleep 2016;39:173-81.

41. Mollayeva T, Thurairajah P, Burton K, et al. The Pittsburgh sleep quality index as a screening tool for sleep dysfunction in clinical and non-clinical samples: a systematic review and meta-analysis. Sleep Med Rev 2016;25:52-73.

42. Germain A, Hall M, Krakow B, et al. A brief sleep scale for posttraumatic stress disorder: Pittsburgh sleep quality index addendum for PTSD. J Anxiety Disord 2005;19:233-44.

43. Insana SP, Hall M, Buysse DJ, et al. Validation of the Pittsburgh Sleep Quality Index Addendum for posttraumatic stress disorder (PSQI-A) in U.S. male military veterans. J Trauma Stress 2013;26:192-200.

44. Nicassio PM, Mendlowitz DR, Fussell JJ, et al. The phenomenology of the pre-sleep state: the development of the pre-sleep arousal scale. Behav Res Ther 1985;23:263-71.

45. Broman J-E, Hetta J. Perceived pre-sleep arousal in patients with persistent psychophysiologic and psychiatric insomnia. Nord $J$ Psychiatry 1994;48:203-7.

46. Robertson JA, Broomfield NM, Espie CA. Prospective comparison of subjective arousal during the pre-sleep period in primary sleep-onset insomnia and normal sleepers. J Sleep Res 2007;16:230-8.

47. Espie CA, Inglis SJ, Harvey L, et al. Insomniacs' attributions. J Psychosom Res 2000;48:141-8. 
48. Edinger JD, Wohlgemuth WK. Psychometric comparisons of the standard and abbreviated DBAS-10 versions of the dysfunctional beliefs and attitudes about sleep questionnaire. Sleep Med 2001;2:493-500.

49. Roenneberg T, Wirz-Justice A, Merrow M. Life between clocks: daily temporal patterns of human chronotypes. J Biol Rhythms 2003;18:80-90.

50. Zavada A, Gordijn MC, Beersma DG, et al. Comparison of the Munich chronotype questionnaire with the Horne-Ostberg's morningness-eveningness score. Chronobiol Int 2005;22:267-78.

51. Levandovski R, Sasso E, Hidalgo MP. Chronotype: a review of the advances, limits and applicability of the main instruments used in the literature to assess human phenotype. Trends Psychiatry Psychother 2013;35:3-11.

52. Cheyne JA. Waterloo unusual sleep experiences questionnaire-VIIla. 2002 http://watarts.uwaterloo.ca/acheyne/spquest01.html (accessed 08 April 2017).

53. Cheyne JA, Newby-Clark IR, Rueffer SD. Relations among hypnagogic and hypnopompic experiences associated with sleep paralysis. J Sleep Res 1999;8:313-7.

54. Denis D, Poerio GL. Terror and bliss? Commonalities and distinctions between sleep paralysis, lucid dreaming, and their associations with waking life experiences. J Sleep Res 2017;26:38-47.

55. Sharpless BA, Doghramji K. Sleep paralysis: historical, psychological, and medical perspectives. New York: Oxford University Press, 2015.

56. Sharpless BA. Exploding head syndrome is common in college students. J Sleep Res 2015;24:447-9.

57. Fulda S, Hornyak M, Müller K, et al. Development and validation of the Munich Parasomnia Screening (MUPS): a questionnaire for parasomnias and nocturnal behaviors. Somnologie 2008;12:56-65

58. Spielberger C, Gorsuch R, Lushene R, et al. Manual for the StateTrait Anxiety Inventory. Palo Alto, CA: Consulting Psychologists Press, 1983.

59. Spielberger CD. State-Trait Anxiety Inventory: bibliography. 2nd edn. Calo Alto, CA: Consulting Psychologists Press, 1989.

60. Angold A, Costello E, Messer S, et al. Development of a short questionnaire for use in epidemiological studies of depression in children and adolescents. Int J Methods Psychiatr Res 1995;5:237-49.

61. American Psychiatric Association. Diagnostic and statistical manual of mental health disorders. 5th edn. Washington, DC: American Psychiatric Association, 2013

62. Gregory AM, Agnew-Blais JC, Matthews T, et al. ADHD and sleep quality: longitudinal analyses from childhood to early adulthood in a twin cohort. J Clin Child Adolesc Psychol 2017;46:284-94.

63. Ronald A, Sieradzka D, Cardno AG, et al. Characterization of psychotic experiences in adolescence using the specific psychotic experiences questionnaire: findings from a study of 5000 16-year-old twins. Schizophr Bull 2014;40:868-77.

64. Freeman D, Garety PA, Bebbington PE, et al. Psychological investigation of the structure of paranoia in a non-clinical population. Br J Psychiatry 2005;186:427-35.

65. Bell V, Halligan PW, Ellis HD. The Cardiff Anomalous Perceptions Scale (CAPS): a new validated measure of anomalous perceptual experience. Schizophr Bull 2006;32:366-77.

66. Mason O, Linney Y, Claridge G. Short scales for measuring schizotypy. Schizophr Res 2005;78:293-6.

67. Taylor MJ, Gregory AM, Freeman D, et al. Do sleep disturbances and psychotic-like experiences in adolescence share genetic and environmental influences? J Abnorm Psychol 2015;124:674-84.

68. Lukat J, Margraf J, Lutz R, et al. Psychometric properties of the Positive Mental Health Scale (PMH-scale). BMC Psychol 2016;4:8.

69. Cohen S, Kamarck T, Mermelstein R. A global measure of perceived stress. J Health Soc Behav 1983;24:385-96.

70. Lee E-H. Review of the psychometric evidence of the perceived stress scale. Asian Nurs Res 2012;6:121-7.

71. Brugha $T$, Bebbington $P$, Tennant $C$, et al. The list of threatening experiences: a subset of 12 life event categories with considerable long-term contextual threat. Psychol Med 1985;15:189-94.

72. Coddington R. Life events scale for children and adolescents: measuring the stressfulness of a child's environment. In: Stress in childhood. New York: AMS Press, 1983.

73. Brugha TS, Cragg D. The list of threatening experiences: the reliability and validity of a brief life events questionnaire. Acta Psychiatr Scand 1990;82:77-81.

74. McAdams TA, Gregory AM, Rowe R, et al. The Genesis 12-19 (G1219) Study: a twin and sibling study of gene-environment interplay and adolescent development in the UK. Twin Res Hum Genet 2013:16:134-43.

75. Currie C, Molcho M, Boyce W, et al. Researching health inequalities in adolescents: the development of the Health Behaviour in School-Aged Children (HBSC) family affluence scale. Soc Sci Med 2008;66:1429-36.

76. Kendler KS, Prescott CA. Caffeine intake, tolerance, and withdrawal in women: a population-based twin study. Am J Psychiatry 1999;156:223-8.

77. Foulds J, Veldheer S, Yingst J, et al. Development of a questionnaire for assessing dependence on electronic cigarettes among a large sample of ex-smoking E-cigarette users. Nicotine Tob Res 2015;17:186-92.

78. Etter JF, Bullen C. Electronic cigarette: users profile, utilization, satisfaction and perceived efficacy. Addiction 2011;106:2017-28.

79. Etter JF. Electronic cigarettes: a survey of users. BMC Public Health 2010;10:231.

80. Magnusson K. Interpreting Cohen's d effect size: R Psychologist. 